\title{
Padovan numbers as sums over partitions into odd parts
}

\section{Cristina Ballantine ${ }^{1 *}$ and Mircea Merca ${ }^{2}$}

\section{"Correspondence:}

cballant@holycross.edu

${ }^{1}$ Department of Mathematics and Computer Science, College of The

Holy Cross, Worcester, MA 01610, USA

Full list of author information is available at the end of the article

\begin{abstract}
Recently it was shown that the Fibonacci numbers can be expressed in terms of multinomial coefficients as sums over integer partitions into odd parts. In this paper, we introduce a similar representation for the Padovan numbers. As a corollary, we derive an infinite family of double inequalities.
\end{abstract}

MSC: Primary 05A20; secondary 05A17

Keywords: integer partitions; Padovan numbers; multinomial coefficients

\section{Introduction}

Integer sequences appear often in many branches of science. One famous example is the Fibonacci numbers that have been known for more than two thousand years and find applications in mathematics, biology, economics, computer science, physics, engineering, architecture, and so forth [1,2]. See also all volumes in the same conference series as [2]. Recall [3] that the sequence $\left\{F_{n}\right\}_{n \geq 0}$ of Fibonacci numbers is defined by the recurrence relation

$$
F_{n}-F_{n-1}-F_{n-2}=0
$$

with seed values

$$
F_{0}=0 \quad \text { and } \quad F_{1}=1
$$

Padovan numbers [4] are much younger. They are named after the mathematician Richard Padovan who attributed their discovery to Dutch architect Dom Hans van der Laan in his 1994 essay Dom Hans van der Laan: Modern Primitive. The Padovan sequence is the sequence of integers $P_{n}$ defined by the initial values

$$
P_{0}=1, \quad P_{1}=0, \quad P_{2}=0,
$$

and the recurrence relation

$$
P_{n}-P_{n-2}-P_{n-3}=0 \text {. }
$$

(C) 2015 Ballantine and Merca. This article is distributed under the terms of the Creative Commons Attribution 4.0 International License (http://creativecommons.org/licenses/by/4.0/), which permits unrestricted use, distribution, and reproduction in any medium, provided you give appropriate credit to the original author(s) and the source, provide a link to the Creative Commons license, and indicate if changes were made. 
The first few terms of the sequence defined by this recurrence relation are:

$1,0,0,1,0,1,1,1,2,2,3,4,5,7,9,12,16,21,28,37, \ldots$ (sequence A000931 in [5]).

Other sources may start the Padovan sequence at a different place. See for example Stewart's paper [6], where this sequence starts with $P_{0}=P_{1}=P_{2}=1$.

It is well known that the ratio of consecutive Fibonacci numbers tends to the golden ratio $\varphi$, i.e.,

$$
\varphi=\lim _{n \rightarrow \infty} \frac{F_{n+1}}{F_{n}}=\frac{1+\sqrt{5}}{2}=1.6180339887498948482 \cdots .
$$

As with the Fibonacci sequence, the ratio of successive Padovan numbers has a limiting value as $n$ tends to infinity. This value is called the plastic number $\rho$,

$$
\rho=\lim _{n \rightarrow \infty} \frac{P_{n+1}}{P_{n}}=\sqrt[3]{\frac{1}{2}+\frac{1}{6} \sqrt{\frac{23}{3}}}+\sqrt[3]{\frac{1}{2}-\frac{1}{6} \sqrt{\frac{23}{3}}}=1.3247179572447460259 \ldots,
$$

which is the unique real solution of the cubic equation

$$
x^{3}-x-1=0
$$

A partition of a positive integer $n$ is a non-increasing sequence of positive integers whose sum is $n$. The terms of the sequence are called the parts of the partition.

Recently, Merca [7], Corollary 9, proved that the Fibonacci numbers can be expressed in terms of multinomial coefficients as sums over integer partitions into odd parts, i.e.,

$$
F_{n}=\sum_{t_{1} a_{1}+t_{2} a_{2}+\cdots+t_{\lceil n / 2\rceil} a_{\lceil n / 2\rceil}=n}\left(\begin{array}{c}
t_{1}+t_{2}+\cdots+t_{\lceil n / 2\rceil} \\
t_{1}, t_{2}, \ldots, t_{\lceil n / 2\rceil}
\end{array}\right), \quad n>0,
$$

where $a_{k}=2 k-1$ and $\lceil x\rceil$ stands for the smallest integer not less than $x$. Because $\rho$ is smaller than $\varphi$, the Padovan sequence increases much slower than the Fibonacci sequence. Nevertheless, the $n$th Padovan number can be expressed in a similar way as a sum of multinomial coefficients over integer partitions of $n$ into odd parts. The difference is given by the fact that these partitions have no part equal to 1 .

Theorem 1 For $n>0$,

$$
P_{n}=\sum_{t_{2} a_{2}+t_{3} a_{3}+\cdots+t_{\lceil n / 2\rceil} a_{\lceil n / 2\rceil}=n}\left(\begin{array}{c}
t_{2}+t_{3}+\cdots+t_{\lceil n / 2\rceil} \\
t_{2}, t_{3}, \ldots, t_{\lceil n / 2\rceil}
\end{array}\right)
$$

where $a_{k}=2 k-1$.

Proof From (2) it follows easily that

$$
P_{n}-P_{n-3}-P_{n-5}-P_{n-7}-\cdots=\delta_{0, n},
$$


where $\delta_{i, j}$ is the Kronecker delta. Then the statement of the theorem follows directly from [7], Theorem 1.

By Theorem 1 it is clear that the number of integer partitions of $n$ into odd parts greater than 1 is less than or equal to the $n$th Padovan number, for any positive integer $n$. As seen in $[8,9]$, this inequality can be improved by exploring the number of multinomial coefficients over integer partitions into odd parts greater than 1 . As a consequence of these facts, a family of double inequalities will be introduced in the paper.

We note that the inequalities proved in this article are, in fact, analytic statements. However, to our knowledge, there are no analytic proofs for these types of results.

\section{On the multinomial coefficients over the partitions into odd parts greater than 1}

In [8], $Q_{k}(n)$ denotes the number of multinomial coefficients satisfying

$$
\left(\begin{array}{c}
t_{1}+t_{2}+\cdots+t_{\lceil n / 2\rceil} \\
t_{1}, t_{2}, \ldots, t_{\lceil n / 2\rceil}
\end{array}\right)=k
$$

and

$$
\sum_{k=1}^{\lceil n / 2\rceil}(2 k-1) t_{k}=n .
$$

In this section, we denote by $Q_{k}^{\prime}(n)$ the number of multinomial coefficients such that

$$
\left(\begin{array}{c}
t_{2}+t_{3}+\cdots+t_{\lceil n / 2\rceil} \\
t_{2}, t_{3}, \ldots, t_{\lceil n / 2\rceil}
\end{array}\right)=k
$$

and

$$
\sum_{k=2}^{\lceil n / 2\rceil}(2 k-1) t_{k}=n
$$

Denoting by $q^{\prime}(n)$ the number of integer partitions of $n$ into odd parts greater than 1 , it is clear that

$$
\sum_{k \geq 1} Q_{k}^{\prime}(n)=q^{\prime}(n)
$$

and

$$
\sum_{k \geq 1} k Q_{k}^{\prime}(n)=P_{n}
$$

We use the convention that $q^{\prime}(0)=1$ and $q^{\prime}(n)=0$ for any negative integer $n$.

Similar to [9], Theorem 2, we obtain the following. 
Theorem 2 For $n, k>0$,

$$
q^{\prime}(n) \leq \frac{1}{k}\left(P_{n}+\sum_{j=1}^{k-1}(k-j) Q_{j}^{\prime}(n)\right)
$$

Next, we relate $Q_{k}(n)$ and $Q_{k}^{\prime}(n)$ in order to derive the generating function for $Q_{k}^{\prime}(n)$ if $k=1$ or a prime power.

Proposition 1 If $k$ is a prime power greater than 2 and $Q_{k}(n)>0$ then

$$
Q_{k}^{\prime}(n)=Q_{k}(n)-1-\left\lfloor\frac{n-1}{k-1}\right\rfloor \bmod 2 \cdot \delta_{0,(n-1) \bmod (k-1)},
$$

where $\lfloor x\rfloor$ stands for the greatest integer less than or equal to $x$.

Proof Let $M_{k}(n)$ denote the number of multinomial coefficients satisfying

$$
\left(\begin{array}{c}
k_{1}+k_{2}+\cdots+k_{n} \\
k_{1}, k_{2}, \ldots, k_{n}
\end{array}\right)=k \text { and } \quad k_{1}+2 k_{2}+\cdots+n k_{n}=n
$$

In [10], Guo showed that $M_{p^{r}}$ (n) equals to the number of positive integer solutions $(x, y)$ of the equation

$$
\left(p^{r}-1\right) x+y=n
$$

satisfying $x \neq y$.

By the same argument, $Q_{p^{r}}(n)$ equals to the number of positive integer solutions $(x, y)$ of (7) with $x, y$ odd and $x \neq y$. Moreover, $Q_{p^{r}}^{\prime}(n)$ equals to the number of positive integer solutions $(x, y)$ of (7) with $x, y$ odd, $x, y>1$, and $x \neq y$.

Note that if $Q_{p^{r}}(n)>0$, then $(1, y)$ with $y$ odd is a solution to (7). In addition, $(x, 1)$ with $x$ odd is a solution to (7) if and only if $p^{r}-1$ divides $n-1$. Thus, (6) follows.

Considering relation (6) and the closed form of the generating functions for $Q_{1}(n)$ and $Q_{2}(n)[8]$, for $|x|<1$ we obtain

$$
\sum_{n=1}^{\infty} Q_{1}^{\prime}(n) x^{n}=\sum_{n=1}^{\infty}\left(\frac{x^{n}}{1-x^{2 n}}-x^{n}\right)=\sum_{n=1}^{\infty} \frac{x^{3 n}}{1-x^{2 n}}
$$

and

$$
\begin{aligned}
\sum_{n=1}^{\infty} Q_{2}^{\prime}(n) x^{n} & =\frac{x^{4}}{\left(1-x^{2}\right)\left(1-x^{4}\right)}-\sum_{n=2}^{\infty} x^{2 n} \\
& =\frac{x^{4}}{\left(1-x^{2}\right)\left(1-x^{4}\right)}-\frac{x^{4}}{1-x^{2}} \\
& =\frac{x^{8}}{\left(1-x^{2}\right)\left(1-x^{4}\right)} .
\end{aligned}
$$

On the other hand, by [8], Theorem 2, it is not difficult to derive the following. 
Theorem 3 Let $k$ be a prime power. For $k>2,|x|<1$,

$$
\sum_{n=1}^{\infty} Q_{k}^{\prime}(n) x^{n}=\frac{x^{k}}{\left(1-x^{2}\right)\left(1-x^{2(k-1)}\right)}-\frac{x^{k}}{1-x^{2 k}}-\frac{x^{k+2}}{1-x^{2}}-\frac{x^{3 k-2}}{1-x^{2(k-1)}} .
$$

\section{A family of double inequalities}

In this section, we denote by $a_{n}$ the $n$th positive integer that is not a power of 2 , i.e.,

$$
\left\{a_{n}\right\}_{n \geq 1}=\{3,5,6,7,9,10,11,12,13,14,15,17,19,20,21,22,23, \ldots\} .
$$

According to [5], A057716, we have

$$
a_{n}=\left\lfloor n+1+\log _{2}\left\lfloor n+1+\log _{2}(n+1)\right\rfloor\right\rfloor .
$$

Theorem 4 Let $k$ be a positive integer. For $0<x<1 / \rho$,

$$
\prod_{n=1}^{\infty}\left(1+x^{a_{n}}\right)<1+B_{k}(x)+\frac{k-1}{k} \sum_{n=1}^{\infty} \frac{x^{3 n}}{1-x^{2 n}}
$$

with

$$
B_{1}(x)=\frac{x^{3}}{1-x^{2}-x^{3}}, \quad B_{2}(x)=\frac{1}{2} \cdot \frac{x^{3}}{1-x^{2}-x^{3}},
$$

and

$$
k B_{k}(x)=(k-1) B_{k-1}(x)+\sum_{j=2}^{k-1} G_{j}(x),
$$

where $G_{j}(x)$ is the generating function for $Q_{j}^{\prime}(n)$.

Proof According to [5], A087897, the generating function for $q^{\prime}(n)$ is given by

$$
\sum_{n=0}^{\infty} q^{\prime}(n) x^{n}=\prod_{n=1}^{\infty}\left(1+x^{a_{n}}\right), \quad|x|<1 .
$$

On the other hand, for $|x|<1 / \rho$ it is well known [5], A000931, that the generating function of the Padovan sequence has the closed form

$$
\sum_{n=0}^{\infty} P_{n} x^{n}=\frac{1-x^{2}}{1-x^{2}-x^{3}}
$$

Then, using equation (8), it follows easily from Theorem 2 that

$$
\prod_{n=1}^{\infty}\left(1+x^{a_{n}}\right) \leq 1+B_{k}(x)+\frac{k-1}{k} \sum_{n=1}^{\infty} \frac{x^{3 n}}{1-x^{2 n}}
$$

Equation (5) implies that the inequality is strict. 
In fact, Theorem 2 and relation (5), imply that the sequence $\left\{B_{k}(x)\right\}_{k>0}$ is strictly monotonically decreasing for $0<x<1 / \rho$. A few special cases of Theorem 4 can easily be derived. So for $k \in\{1,2,3\}$, we obtain

$$
\begin{aligned}
\prod_{n=1}^{\infty}\left(1+x^{a_{n}}\right) & <1+\frac{1}{3} \cdot \frac{x^{3}}{1-x^{2}-x^{3}}+\frac{1}{3} \cdot \frac{x^{8}}{\left(1-x^{2}\right)\left(1-x^{4}\right)}+\frac{2}{3} \sum_{n=1}^{\infty} \frac{x^{3 n}}{1-x^{2 n}} \\
& <1+\frac{1}{2} \cdot \frac{x^{3}}{1-x^{2}-x^{3}}+\frac{1}{2} \sum_{n=1}^{\infty} \frac{x^{3 n}}{1-x^{2 n}} \\
& <\frac{1-x^{2}}{1-x^{2}-x^{3}}, \quad 0<x<1 / \rho .
\end{aligned}
$$

In the cases $k \in\{4,5,6\}$ the inequalities are more involved. It is still an open problem to give the expression of the generating function for $Q_{k}^{\prime}(n)$ when $k$ is a positive integer that is not a prime power. Replacing $x$ by $1 / 4$ in the last inequalities, we get the following inequalities:

$$
\begin{aligned}
\prod_{n=1}^{\infty}\left(1+\frac{1}{4^{a_{n}}}\right) & <1+\frac{61,259}{10,832,400}+\frac{2}{3} \sum_{n=1}^{\infty} \frac{1}{4^{3 n}-4^{n}} \\
& <1+\frac{1}{118}+\frac{1}{2} \sum_{n=1}^{\infty} \frac{1}{4^{3 n}-4^{n}} \\
& <1+\frac{1}{59}=1.016949153 \cdots
\end{aligned}
$$

Theorem 5 Let $k$ be a positive integer. For $0<x<1$,

$$
A_{k}(x)+\sum_{n=1}^{\infty} \frac{x^{3 n}}{1-x^{2 n}}<\prod_{n=1}^{\infty}\left(1+x^{a_{n}}\right)
$$

with

$$
A_{1}(x)=1, \quad A_{2}(x)=1+\frac{x^{8}}{\left(1-x^{2}\right)\left(1-x^{4}\right)},
$$

and, for $k \geq 3$,

$$
A_{k}(x)=A_{k-1}(x)+\frac{x^{p_{k}}}{\left(1-x^{2}\right)\left(1-x^{2\left(p_{k}-1\right)}\right)}-\frac{x^{p_{k}}}{1-x^{2 p_{k}}}-\frac{x^{p_{k}+2}}{1-x^{2}}-\frac{x^{3 p_{k}-2}}{1-x^{2\left(p_{k}-1\right)}},
$$

where $p_{k}$ is the kth positive integer that is a prime power.

Proof By (4), we have the inequality

$$
\sum_{j=1}^{k} Q_{p_{j}}^{\prime}(n) \leq q^{\prime}(n), \quad n>0 .
$$


For $0<x<1$, from (8), (9), and (10), we can write

$$
1+\frac{x^{8}}{\left(1-x^{2}\right)\left(1-x^{4}\right)}+\sum_{j=2}^{k} G_{j}(x)+\sum_{n=1}^{\infty} \frac{x^{3 n}}{1-x^{2 n}}<\prod_{n=1}^{\infty}\left(1+x^{a_{n}}\right)
$$

where $G_{j}(x)$ is the generating function for $Q_{p_{j}}^{\prime}(n)$. The proof follows easily considering Theorem 3 .

The cases $k \in\{1,2\}$ of Theorem 5 can be written as

$$
\begin{aligned}
\prod_{n=1}^{\infty}\left(1+x^{a_{n}}\right) & >1+\frac{x^{8}}{\left(1-x^{2}\right)\left(1-x^{4}\right)}+\sum_{n=1}^{\infty} \frac{x^{3 n}}{1-x^{2 n}} \\
& >1+\sum_{n=1}^{\infty} \frac{x^{3 n}}{1-x^{2 n}}, \quad 0<x<1 .
\end{aligned}
$$

For $k>2$ the inequalities are more involved. Replacing $x$ by $1 / 4$, we obtain

$$
\begin{aligned}
\prod_{n=1}^{\infty}\left(1+\frac{1}{4^{a_{n}}}\right) & >1+\frac{1}{61,200}+\sum_{n=1}^{\infty} \frac{1}{4^{3 n}-4^{n}} \\
& >1+\sum_{n=1}^{\infty} \frac{1}{4^{3 n}-4^{n}} .
\end{aligned}
$$

For $0<x<1$, we remark that the sequence $\left\{A_{k}(x)\right\}_{k>0}$ is strictly monotonically increasing. In addition, Theorems 4 and 5 can be written as follows.

Theorem 6 Let $k_{1}$ and $k_{2}$ be positive integers. For $0<x<1 / \rho$,

$$
A_{k_{1}}(x)+\sum_{n=1}^{\infty} \frac{x^{3 n}}{1-x^{2 n}}<\prod_{n=1}^{\infty}\left(1+x^{a_{n}}\right)<1+B_{k_{2}}(x)+\frac{k_{2}-1}{k_{2}} \sum_{n=1}^{\infty} \frac{x^{3 n}}{1-x^{2 n}}
$$

The case $k_{1}=k_{2}=3$ and $x=1 / 4$ of this theorem reads

$$
1+\frac{37}{2,227,680}+\sum_{n=1}^{\infty} \frac{1}{4^{3 n}-4^{n}}<\prod_{n=1}^{\infty}\left(1+\frac{1}{4^{a_{n}}}\right)<1+\frac{61,259}{10,832,400}+\frac{2}{3} \sum_{n=1}^{\infty} \frac{1}{4^{3 n}-4^{n}}
$$

The following inequality follows directly from Theorem 6.

Corollary 1 Let $k$ be a positive integer. For $0<x<1 / \rho$,

$$
\sum_{n=1}^{\infty} \frac{x^{3 n}}{1-x^{2 n}}<k\left(1+B_{k}(x)-A_{k}(x)\right)
$$

Many special cases of this corollary can easily be derived. For instance, considering the case $k=1$ and $x=1 / 4$ of this corollary, we obtain

$$
\frac{1}{60}<\sum_{n=1}^{\infty} \frac{1}{4^{3 n}-4^{n}}<\frac{1}{59}
$$


For $k=2$ and $x=1 / 4$, we have

$$
\frac{1}{60}+\frac{1}{4,080}<\sum_{n=1}^{\infty} \frac{1}{4^{3 n}-4^{n}}<\frac{1}{59}-\frac{1}{30,600}
$$

Another family of upper bounds for $\sum_{n=1}^{\infty} x^{3 n} /\left(1-x^{2 n}\right)$ can easily be derived by (5) if we use Theorem 3 .

Theorem 7 Let $k$ be a positive integer. For $0<x<1 / \rho$,

$$
\sum_{n=1}^{\infty} \frac{x^{3 n}}{1-x^{2 n}}<C_{k}(x)
$$

with

$$
C_{1}(x)=\frac{x^{3}}{1-x^{2}-x^{3}}, \quad C_{2}(x)=C_{1}(x)-\frac{2 x^{8}}{\left(1-x^{2}\right)\left(1-x^{4}\right)},
$$

and

$$
C_{k}(x)=C_{k-1}(x)-\frac{p_{k} x^{p_{k}}}{\left(1-x^{2}\right)\left(1-x^{2\left(p_{k}-1\right)}\right)}+\frac{p_{k} x^{p_{k}}}{1-x^{2 p_{k}}}+\frac{p_{k} x^{p_{k}+2}}{1-x^{2}}+\frac{p_{k} x^{3 p_{k}-2}}{1-x^{2\left(p_{k}-1\right)}}
$$

where $p_{k}$ is the kth positive integer that is a prime power.

Proof According to (5), we have the inequality

$$
\sum_{j=1}^{p_{k}} p_{j} Q_{p_{j}}^{\prime}(n) \leq P_{n}, \quad n>0
$$

Using equations (8) and (9), the generating function of the Padovan sequence, and Theorem 3 , we arrive at our conclusion.

The sequence $\left\{C_{k}(x)\right\}_{k>0}$ is strictly monotonically decreasing when $0<x<1 / \rho$. For $k \in$ $\{1,2,3,4,5\}$, we note the identity

$$
C_{k}(x)=k\left(1+B_{k}(x)-A_{k}(x)\right), \quad 0<x<1 / \rho .
$$

\section{Other inequalities}

By (2), it is an easy exercise to derive the following identity:

$$
\sum_{j=0}^{k}\left(\begin{array}{l}
k \\
j
\end{array}\right) P_{n-2 k-j}=P_{n}
$$

where $n, k$ are a non-negative integers such that $n \geq 3 k$. Then, Theorem 2 (with $k=1$ ) implies that, for $n \geq 3 k$, we have

$$
\sum_{j=0}^{k}\left(\begin{array}{l}
k \\
j
\end{array}\right) q^{\prime}(n-2 k-j) \leq P_{n}
$$


The next theorem introduces an infinite family of upper bounds for the generating function of the number of partitions into odd parts greater than 1 .

Theorem 8 Let $k$ be a positive integer. For $0<x<1 / \rho$,

$$
\prod_{n=1}^{\infty}\left(1+x^{a_{n}}\right)<\frac{1-x^{2}}{x^{2 k}(1+x)^{k}\left(1-x^{2}-x^{3}\right)}-\frac{1}{x^{2 k}(1+x)^{k}} \sum_{n=0}^{3 k-1}\left(P_{n}-S_{n, k}\right) x^{n}
$$

where

$$
S_{n, k}=\sum_{j=0}^{k}\left(\begin{array}{l}
k \\
j
\end{array}\right) q^{\prime}(n-2 k-j)
$$

Proof We first give the generating function for $S_{n, k}$. We have

$$
\begin{aligned}
\sum_{n=0}^{\infty} S_{n, k} x^{n} & =\sum_{n=0}^{\infty}\left(\sum_{j=0}^{k}\left(\begin{array}{l}
k \\
j
\end{array}\right) q^{\prime}(n-2 k-j)\right) x^{n} \\
& =\left(\sum_{j=0}^{k}\left(\begin{array}{l}
k \\
j
\end{array}\right) x^{j}\right)\left(\sum_{n=2 k}^{\infty} q^{\prime}(n-2 k) x^{n}\right) .
\end{aligned}
$$

Therefore,

$$
\sum_{n=0}^{\infty} S_{n, k} x^{n}=x^{2 k}(1+x)^{k} \prod_{n=1}^{\infty}\left(1+x^{a_{n}}\right), \quad|x|<1
$$

From (16), for $x>0$, we have

$$
\sum_{n=3 k}^{\infty} S_{n, k} x^{n}<\sum_{n=3 k}^{\infty} P_{n} x^{n}
$$

Thus, for $0<x<1 / \rho$,

$$
x^{2 k}(1+x)^{k} \prod_{n=1}^{\infty}\left(1+x^{a_{n}}\right)-\sum_{n=0}^{3 k-1} S_{n, k} x^{n}<\frac{1-x^{2}}{1-x^{2}-x^{3}}-\sum_{n=0}^{3 k-1} P_{n} x^{n}
$$

and the statement of the theorem follows.

It can easily be checked that, for $k<9$, the inequality in Theorem 8 reduces to the inequality (12). When $k=9$ and $0<x<1 / \rho$, Theorem 8 gives the stronger inequality

$$
\begin{aligned}
\prod_{n=1}^{\infty}\left(1+x^{a_{n}}\right)< & \frac{1+9 x+35 x^{2}+75 x^{3}+90 x^{4}+42 x^{5}}{(1+x)^{9}\left(1-x^{2}-x^{3}\right)} \\
& -\frac{42 x^{6}+90 x^{7}+76 x^{8}+35 x^{9}+8 x^{10}}{(1+x)^{9}\left(1-x^{2}-x^{3}\right)} .
\end{aligned}
$$


Setting $x=1 / 4$, we obtain

$$
\prod_{n=1}^{\infty}\left(1+\frac{1}{4^{a_{n}}}\right)<\frac{117,187,264}{115,234,375}=1.016947105 \cdots
$$

which is an improvement to the inequality (14). In addition, we remark that a lower bound for this product is given by

$$
\prod_{n=1}^{8}\left(1+\frac{1}{4^{a_{n}}}\right)=1.016932255 \cdots
$$

Theorem 8 gives a decreasing sequence of upper bounds for the generating function of the number of partitions into odd parts greater than 1 . To see this, let

$$
R_{k}(x)=\frac{1-x^{2}}{x^{2 k}(1+x)^{k}\left(1-x^{2}-x^{3}\right)}-\frac{1}{x^{2 k}(1+x)^{k}} \sum_{n=0}^{3 k-1}\left(P_{n}-S_{n, k}\right) x^{n} .
$$

Then, for $x>0$, the inequality $R_{k+1}(x)-R_{k}(x) \leq 0$ is equivalent to

$$
1-x^{2}+x^{2}(1+x) \sum_{n=0}^{3 k-1}\left(P_{n}-S_{n, k}\right) x^{n}-\sum_{n=0}^{3 k+2}\left(P_{n}-S_{n, k+1}\right) x^{n} \leq 0 .
$$

That $\left\{R_{k}(x)\right\}_{k \geq 0}$ is decreasing follows from the identity

$$
S_{n+3, k+1}=S_{n+1, k}+S_{n, k}
$$

and the inequality (16). The identity (17) is easily deduced from the definition of $S_{n, k}$. As in [8], Theorem 7, we have the following.

Theorem 9 Let $k$ be a non-negative integer. For $0<x<1 / \rho$,

$$
\begin{aligned}
& 2 \prod_{n=1}^{\infty}\left(1+x^{a_{n}}\right)-\sum_{n=1}^{\infty} \frac{x^{3 n}}{1-x^{2 n}} \\
& \quad<\frac{1-x^{2}}{x^{2 k}(1+x)^{k}\left(1-x^{2}-x^{3}\right)}-\frac{1}{x^{2 k}(1+x)^{k}} \sum_{n=0}^{3 k}\left(P_{n}-T_{n, k}\right) x^{n}
\end{aligned}
$$

where

$$
T_{n, k}=2 \sum_{j=0}^{k}\left(\begin{array}{l}
k \\
j
\end{array}\right) q^{\prime}(n-2 k-j)-\sum_{j=0}^{k}\left(\begin{array}{l}
k \\
j
\end{array}\right) Q_{1}^{\prime}(n-2 k-j),
$$

with $Q_{1}^{\prime}(n)=0$ for any non-positive $n$.

Proof Considering the identity (15) and the case $k=2$ of Theorem 2,

$$
2 q^{\prime}(n)-Q_{1}^{\prime}(n) \leq P(n), \quad n>0,
$$

the proof is similar to the proof of Theorem 8 . 
It is not difficult to check that, for $k<11$, Theorem 9 reduces to inequality (11). If $k=11$ and $0<x<1 / \rho$ then we obtain the inequality

$$
\begin{aligned}
& 2 \prod_{n=1}^{\infty}\left(1+x^{a_{n}}\right)-\sum_{n=1}^{\infty} \frac{x^{3 n}}{1-x^{2 n}} \\
& <\frac{2+22 x+108 x^{2}+307 x^{3}+539 x^{4}+539 x^{5}+99 x^{6}}{(1+x)^{11}\left(1-x^{2}-x^{3}\right)} \\
& \quad-\frac{594 x^{7}+1,056 x^{8}+1,012 x^{9}+638 x^{10}+274 x^{11}+77 x^{12}+12 x^{13}}{(1+x)^{11}\left(1-x^{2}-x^{3}\right)} .
\end{aligned}
$$

From this inequality, with $x$ replaced by $1 / 4$, we obtain

$$
\prod_{n=1}^{\infty}\left(1+\frac{1}{4^{a_{n}}}\right)<1+\frac{24,414,033}{2,880,859,375}+\frac{1}{2} \sum_{n=1}^{\infty} \frac{1}{4^{3 n}-4^{n}} .
$$

This is an improved version of the inequality (13).

One can similarly consider the cases $k=3,4,5$ in Theorem 2 to obtain stronger but more complicated inequalities involving the generating function for the number of partitions into odd parts greater than 1 and the generating function for the number of odd divisors greater than 1.

\section{Results and discussion}

According to Theorem 1, the $n$th Padovan number can be expressed as a sum of multinomial coefficients over integer partitions of $n$ into odd parts greater than 1 . This result allows us to derive a family of inequalities (see Theorem 2) that involve the Padovan number $P_{n}$ and the number of partitions of $n$ into odd parts greater than 1, where the trivial inequality

$$
q^{\prime}(n) \leq P_{n}
$$

is the first entry. The second entry provides a better inequality

$$
q^{\prime}(n) \leq \frac{1}{2}\left(P_{n}+\tau_{o}(n)-1\right)
$$

but it involves, in addition, the number of positive odd divisors function

$$
\tau_{o}(n)=\sum_{\substack{d \mid n \\ d \text { odd }}} 1
$$

This form of the second entry follows from (8) and the well-known generating function of $\tau_{o}(n)$,

$$
\sum_{n=1}^{\infty} \tau_{o}(n)=\sum_{n=1}^{\infty} \frac{x^{n}}{1-x^{2 n}}, \quad|x|<1
$$


Moreover, using the generating function of $\lfloor n / k\rfloor$,

$$
\sum_{n=0}^{\infty}\left\lfloor\frac{n}{k}\right\rfloor x^{n}=\frac{x^{k}}{(1-x)\left(1-x^{k}\right)}, \quad|x|<1,
$$

for $n>2$, we deduce that

$$
Q_{2}^{\prime}(n)=\frac{1+(-1)^{n}}{2}\left(\left\lfloor\frac{n}{4}\right\rfloor-1\right) .
$$

Thus, the third entry in the family of inequalities can be written as

$$
q^{\prime}(n) \leq \frac{1}{3} P_{n}+\frac{2}{3}\left(\tau_{o}(n)-1\right)+\frac{1+(-1)^{n}}{6}\left(\left\lfloor\frac{n}{4}\right\rfloor-1\right), \quad n>2 .
$$

We now use Theorem 3 to derive a simple expression for $Q_{k}^{\prime}(n)$ when $k$ is a prime power greater than 2 .

Corollary 2 Let $k$ be a prime power. For $k>2$ and $n \geq 3 k-2$,

$$
Q_{k}^{\prime}(n)=\frac{1+(-1)^{n+k}}{2}\left\lfloor\frac{n+k-3}{2 k-2}\right\rfloor-\delta_{0,(n-k) \bmod 2 k}-\delta_{0,(n-k) \bmod 2},
$$

where $\delta_{i, j}$ is the Kronecker delta.

Proof From (18), with $x$ replaced by $x^{2}$ and $k$ replaced by $k-1$, we obtain

$$
\sum_{n=0}^{\infty} \frac{1+(-1)^{n+k}}{2}\left\lfloor\frac{n+k-2}{2(k-1)}\right\rfloor x^{n}=\frac{x^{k}}{\left(1-x^{2}\right)\left(1-x^{2(k-1)}\right)}, \quad|x|<1 .
$$

On the other hand, since

$$
\frac{1}{1-x^{k}}=\sum_{n=0}^{\infty} x^{k n}, \quad|x|<1
$$

we derive

$$
\begin{array}{ll}
\frac{x^{k}}{1-x^{2 k}}=\sum_{n=k}^{\infty} \delta_{0,(n-k) \bmod 2 k} \cdot x^{n}, & |x|<1, \\
\frac{x^{k+2}}{1-x^{2}}=\sum_{n=k+2}^{\infty} \delta_{0,(n-k) \bmod 2} \cdot x^{n}, & |x|<1,
\end{array}
$$

and

$$
\begin{aligned}
\frac{x^{3 k-2}}{1-x^{2(k-1)}} & =\sum_{n=3 k-2}^{\infty} \delta_{0,(n-3 k+2) \bmod (2 k-2)} \cdot x^{n} \\
& =\sum_{n=3 k-2}^{\infty} \frac{1+(-1)^{n+k}}{2} \cdot \delta_{0,(n-3 k+2) \bmod (2 k-2)} \cdot x^{n}
\end{aligned}
$$




$$
\begin{aligned}
& =\sum_{n=3 k-2}^{\infty} \frac{1+(-1)^{n+k}}{2}\left(\left\lfloor\frac{n-3 k+2}{2 k-2}\right\rfloor-\left\lfloor\frac{n-1-3 k-2}{2 k-2}\right\rfloor\right) x^{n} \\
& =\sum_{n=3 k-2}^{\infty} \frac{1+(-1)^{n+k}}{2}\left(\left\lfloor\frac{n+k-2}{2 k-2}\right\rfloor-\left\lfloor\frac{n+k-3}{2 k-2}\right\rfloor\right) x^{n}, \quad|x|<1,
\end{aligned}
$$

where we have invoked

$$
\delta_{0, n \bmod k}=\left\lfloor\frac{n}{k}\right\rfloor-\left\lfloor\frac{n-1}{k}\right\rfloor
$$

The proof follows easily from Theorem 3.

The case $k=3$ of this corollary is given by

$$
Q_{3}^{\prime}(n)=\frac{1-(-1)^{n}}{2}\left\lfloor\frac{n}{4}\right\rfloor-\delta_{0,(n-3) \bmod 6}-\delta_{0,(n-1) \bmod 2,} \quad n \geq 7
$$

In this context, the case $k=4$ of Theorem 2 can be written as

$$
q^{\prime}(n) \leq \frac{1}{4} P_{n}+\frac{3}{4}\left(\tau_{o}(n)-1\right)+\frac{3+(-1)^{n}}{8}\left\lfloor\frac{n}{4}\right\rfloor-\frac{3-(-1)^{n}}{4}-\delta_{0,(n-3) \bmod 6}, \quad n \geq 7 .
$$

It is still an open problem to give a formula for $Q_{k}^{\prime}(n)$ when $k$ is not a prime power.

\section{Concluding remarks}

A representation for the Padovan number $P_{n}$ as sum of multinomial coefficients over integer partitions of $n$ into odd parts greater than 1 , has been introduced in this paper. For $0<x<1 / \rho$, this result allows us to approximate the infinite product

$$
\prod_{n=1}^{\infty}\left(1+x^{a_{n}}\right)=\prod_{n=1}^{\infty} \frac{1+x^{n}}{1+x^{2^{n-1}}}
$$

by the infinite sum

$$
\sum_{n=1}^{\infty} \frac{x^{3 n}}{1-x^{2 n}}
$$

and vice versa.

We remark that this infinite sum can be replaced by a series which converges much faster for $|x|<1$.

Theorem 10 For $|x|<1$,

$$
\sum_{n=1}^{\infty} \frac{x^{3 n}}{1-x^{2 n}}=\sum_{n=1}^{\infty} \frac{1-x^{4 n+1}}{\left(1-x^{2 n}\right)\left(1-x^{2 n+1}\right)} \cdot x^{2 n^{2}+n}
$$

Proof Due to Wrench, Jr. [11], p.644, solution to exercise 5.2.3-27, we have

$$
\sum_{n=1}^{\infty} \frac{a_{n} q^{n}}{1-q^{n}}=\sum_{n=1}^{\infty}\left(a_{n}+\sum_{k=1}^{\infty}\left(a_{n}+a_{n+k}\right) q^{k n}\right) q^{n^{2}}, \quad|q|<1
$$


By this relation, with $a_{n}$ replaced by $x^{n}$ and $q$ replaced by $x^{2}$, we obtain

$$
\begin{aligned}
\sum_{n=1}^{\infty} \frac{x^{3 n}}{1-x^{2 n}} & =\sum_{n=1}^{\infty}\left(x^{n}+\sum_{k=1}^{\infty}\left(x^{n}+x^{n+k}\right) x^{2 k n}\right) x^{2 n^{2}} \\
& =\sum_{n=1}^{\infty}\left(1+\sum_{k=1}^{\infty} x^{2 n k}+\sum_{k=1}^{\infty} x^{(2 n+1) k}\right) x^{2 n^{2}+n} \\
& =\sum_{n=1}^{\infty}\left(1+\left(\frac{1}{1-x^{2 n}}-1\right)+\left(\frac{1}{1-x^{2 n+1}}-1\right)\right) x^{2 n^{2}+n} \\
& =\sum_{n=1}^{\infty}\left(1+\frac{x^{2 n}}{1-x^{2 n}}+\frac{x^{2 n+1}}{1-x^{2 n+1}}\right) x^{2 n^{2}+n} \\
& =\sum_{n=1}^{\infty} \frac{1-x^{4 n+1}}{\left(1-x^{2 n}\right)\left(1-x^{2 n+1}\right)} \cdot x^{2 n^{2}+n} .
\end{aligned}
$$

This concludes the proof.

Finally, we note that when the problem of finding a closed form for the generating function of $Q_{k}^{\prime}(n)$ for arbitrary $k$ will be solved, then further, stronger inequality families will follow by the methods used in this article.

\section{Competing interests}

The authors declare that they have no competing interests.

\section{Authors' contributions}

Both authors, viz. CB and MM with the consultation of each other, carried out this work and drafted the manuscript together. Both authors read and approved the final manuscript.

\section{Author details}

'Department of Mathematics and Computer Science, College of The Holy Cross, Worcester, MA 01610, USA.

${ }^{2}$ Department of Mathematics, University of Craiova, Craiova, DJ 200585, Romania.

\section{Acknowledgements}

The second author wishes to thank the College of the Holy Cross for its hospitality during the writing of this article. The authors thank the anonymous referees for the careful reading of the manuscript and for their useful comments. This work was partially supported by a grant from the Simons Foundation (\#245997).

Received: 30 September 2015 Accepted: 21 December 2015 Published online: 04 January 2016

\section{References}

1. Garland, TH: Fascinating Fibonaccis: Mystery and Magic in Numbers. Dale Seymour Publications, Palo Alto (1987)

2. Howard, FT: Applications of Fibonacci numbers: Proceedings of the 10th International Research Conference on Fibonacci Numbers and Their Applications Held, Northern Arizona University, Flagstaff, AZ, June 24-28, 2002 , vol. 9. Kluwer Academic, Dordrecht (2004)

3. Koshi, T: Elementary Number Theory with Applications, 2nd edn. Academic Press, New York (2007)

4. Padovan, R: Dom Hans van der Laan: Modern Primitive. Architectura \& Natura Press, Amsterdam (1994)

5. Sloane, NJA: The on-Line Encyclopedia of Integer Sequences (2014). Published electronically at http://oeis.org

6. Stewart, I: Tales of a neglected number. Sci. Am. 274, 102-103 (1996)

7. Merca, M: A generalization of the symmetry between complete and elementary symmetric functions. Indian J. Pure Appl. Math. 45, 75-89 (2014)

8. Ballantine, C, Merca, M: Inequalities involving the generating function for the number of partitions into odd parts (2014) arXiv:1407.5552v2

9. Merca, M: New upper bounds for the number of partitions into a given number of parts. J. Number Theory 142 , 298-304 (2014)

10. Guo, VJW: Proof of a conjecture of Mircea Merca. J. Number Theory 147, 590-593 (2015)

11. Knuth, DE: The art of computer programming. In: Sorting and Searching, 2nd edn., vol. 3. Addison-Wesley, Reading (1997) 